\title{
Fractional operators with exponential kernels and a Lyapunov type inequality
}

Thabet Abdeljawad*

\section{"Correspondence:}

tabdeljawad@psu.edu.sa Department of Mathematics and Physical Sciences, Prince Sultan University, P.O. Box 66833, Riyadh, 11586, Saudi Arabia

\begin{abstract}
In this article, we extend fractional calculus with nonsingular exponential kernels, initiated recently by Caputo and Fabrizio, to higher order. The extension is given to both left and right fractional derivatives and integrals. We prove existence and uniqueness theorems for the Caputo (CFC) and Riemann (CFR) type initial value problems by using Banach contraction theorem. Then we prove Lyapunov type inequality for the Riemann type fractional boundary value problems within the exponential kernels. Illustrative examples are analyzed and an application about Sturm-Liouville eigenvalue problem in the sense of this fractional calculus is given as well.
\end{abstract}

Keywords: CFC fractional derivative; CFR fractional derivative; Lyapunov inequality; boundary value problem; higher order; exponential kernel

\section{Introduction and preliminaries}

Fractional calculus [1-5] has been attractive to many researchers in the last three decades or so. Some researchers have found it necessary to define new fractional derivatives with different singular or nonsingular kernels in order to provide more sufficient area to model more real-world problems in different fields of science and engineering [6, 7]. In [8-12] the authors studied new type of fractional derivatives where the kernel is of exponential type and in $[13,14]$ the authors studied new fractional derivatives with Mittag-Leffler kernels. For the discrete counter parts we refer to the work in [15-18]. In this work we extend the fractional calculus with exponential kernels proposed and studied in $[8,9]$ to higher order, prove some existence and uniqueness theorems and prove Lypanouv type inequalities for boundary value problems in the frame of this calculus. The extension is achieved for both left and right fractional derivatives and integrals so that we prepare for integration by parts in higher order to serve fractional variational calculus in the frame of this calculus $[19,20]$.

Definition 1 ([1]) For $\alpha>0, a \in \mathbb{R}$ and $f$ a real-valued function defined on $[a, \infty)$, the left Riemann Liouville fractional integral is defined by

$$
\left({ }_{a} I^{\alpha} f\right)(t)=\frac{1}{\Gamma(\alpha)} \int_{a}^{t}(t-s)^{\alpha-1} f(s) d s .
$$

(c) The Author(s) 2017. This article is distributed under the terms of the Creative Commons Attribution 4.0 International License (http://creativecommons.org/licenses/by/4.0/), which permits unrestricted use, distribution, and reproduction in any medium, provided you give appropriate credit to the original author(s) and the source, provide a link to the Creative Commons license, and indicate if changes were made. 
This is fractionalizing of the $n$-iterated integral $\left({ }_{a} I^{n} f\right)(t)=\frac{1}{(n-1) !} \int_{a}^{t}(t-s)^{n-1} f(s) d s$. The right fractional integral ending at $b$ is defined by

$$
\left(I_{b}^{\alpha} f\right)(t)=\frac{1}{\Gamma(\alpha)} \int_{t}^{b}(s-t)^{\alpha-1} f(s) d s
$$

Definition 2 ([8,15]) Let $f \in H^{1}(a, b), a<b, \alpha \in[0,1]$, then the definition of the new (left Caputo) fractional derivative in the sense of Caputo and Fabrizio becomes

$$
\left({ }_{a}^{\mathrm{CFC}} D^{\alpha} f\right)(t)=\frac{B(\alpha)}{1-\alpha} \int_{a}^{t} f^{\prime}(x) e^{\left[-\alpha \frac{(t-x)^{\alpha}}{1-\alpha}\right]} d x
$$

and in the left Riemann-Liouville sense has the following form:

$$
\left({ }_{a}^{\mathrm{CFR}} D^{\alpha} f\right)(t)=\frac{B(\alpha)}{1-\alpha} \frac{d}{d t} \int_{a}^{t} f(x) e^{\left[-\alpha \frac{(t-x)^{\alpha}}{1-\alpha}\right]} d x
$$

The associated fractional integral is

$$
\left({ }_{a}^{\mathrm{CF}} I^{\alpha} f\right)(t)=\frac{1-\alpha}{B(\alpha)} f(t)+\frac{\alpha}{B(\alpha)} \int_{a}^{t} f(s) d s
$$

where $B(\alpha)>0$ is a normalization function satisfying $B(0)=B(1)=1$. In the right case we have

$$
\left({ }^{\mathrm{CFC}} D_{b}^{\alpha} f\right)(t)=\frac{-B(\alpha)}{1-\alpha} \int_{t}^{b} f^{\prime}(x) e^{\left[-\alpha \frac{(x-t)^{\alpha}}{1-\alpha}\right]} d x
$$

and in the right Riemann-Liouville sense has the following form:

$$
\left({ }^{\mathrm{CFR}} D_{b}^{\alpha} f\right)(t)=\frac{B(\alpha)}{1-\alpha} \frac{-d}{d t} \int_{t}^{b} f(x) e^{\left[-\alpha \frac{(x-t)^{\alpha}}{1-\alpha}\right]} d x
$$

The associated fractional integral is

$$
\left({ }^{\mathrm{CF}} I_{b}^{\alpha} f\right)(t)=\frac{1-\alpha}{B(\alpha)} f(t)+\frac{\alpha}{B(\alpha)} \int_{t}^{b} f(s) d s
$$

In $[8,15]$, it was verified that $\left({ }_{a}^{\mathrm{CF}} I_{a}^{\alpha C F R} D^{\alpha} f\right)(t)=f(t)$ and $\left({ }_{a}^{\mathrm{CFR}} D_{a}^{\alpha}{ }_{a}^{\mathrm{CF}} I^{\alpha} f\right)(t)=f(t)$. Also, in the right case $\left({ }^{\mathrm{CF}} I_{b}^{\alpha \mathrm{CFR}} D_{b}^{\alpha} f\right)(t)=f(t)$ and $\left({ }^{\mathrm{CFR}} D_{b}^{\alpha \mathrm{CF}} I_{b}^{\alpha} f\right)(t)=f(t)$. From $[8,15]$ we recall the relation between the Riemann-Liouville and Caputo new derivatives as

$$
\left({ }_{a}^{\mathrm{CFC}} D^{\alpha} f\right)(t)=\left({ }_{a}^{\mathrm{CFR}} D^{\alpha} f\right)(t)-\frac{B(\alpha)}{1-\alpha} f(a) e^{-\frac{\alpha}{1-\alpha}(t-a)^{\alpha}} .
$$

In next section, we extend Definition 2 to arbitrary $\alpha>0$.

Lemma 1 ([15]) For $0<\alpha<1$, we have

$$
\left({ }_{a}^{\mathrm{CF}} I_{a}^{\alpha}{ }_{a}^{\mathrm{CFC}} D^{\alpha} f\right)(x)=f(x)-f(a)
$$


and

$$
\left({ }^{\mathrm{CF}} I_{b}^{\alpha \mathrm{CFC}} D_{b}^{\alpha} f\right)(x)=f(x)-f(b)
$$

One of our main purposes in this article is to obtain the corresponding result of the following popular Lyapunov inequality result for CFR boundary value problems.

Theorem 1 ([21]) If the boundary value problem

$$
y^{\prime \prime}(t)+q(t) y(t)=0, \quad t \in(a, b), y(a)=y(b)=0,
$$

has a nontrivial solution, where $q$ is a real continuous function, then

$$
\int_{a}^{b}|q(s)| d s>\frac{4}{b-a}
$$

The generalization of the above Lyapunov inequality to fractional boundary value problems has been the interest of some researchers in the last few years. For examples, we refer the reader to [22-26]. For discrete fractional counterparts of Lyapunov inequalities we refer to [27] and for the $q$-fractional types we refer to [28]. For recent extensions to higher order and Lyapunov type inequalities for fractional operators with Mittag-Leffler kernels and fractional difference operators with discrete exponential kernels we refer to [29] and [30], respectively. For the Lyapnunov inequalities of fractional difference operators with discrete Mittag-Leffer kernels we refer to [31].

\section{The higher order fractional derivatives and integrals}

Definition 3 Let $n<\alpha \leq n+1$ and $f$ be such that $f^{(n)} \in H^{1}(a, b)$. Set $\beta=\alpha-n$. Then $\beta \in(0,1]$ and we define

$$
\left({ }_{a}^{\mathrm{CFC}} \mathbf{D}^{\alpha} f\right)(t)=\left({ }_{a}^{\mathrm{CFC}} D^{\beta} f^{(n)}\right)(t) .
$$

In the left Riemann-Liouville sense has this the following form:

$$
\left({ }_{a}^{\mathrm{CFR}} \mathbf{D}^{\alpha} f\right)(t)=\left({ }_{a}^{\mathrm{CFR}} D^{\beta} f^{(n)}\right)(t) .
$$

The associated fractional integral is

$$
\left({ }_{a}^{\mathrm{CF}} \mathbf{I}^{\alpha} f\right)(t)=\left({ }_{a} I_{a}^{n \mathrm{CF}} I^{\beta} f\right)(t) .
$$

Note that if we use the convention that $\left({ }_{a} I^{0} f\right)(t)=f(t)$ then for the case $0<\alpha \leq 1$ we have $\beta=\alpha$ and hence $\left({ }_{a} I^{\alpha} f\right)(t)=\left({ }_{a} I^{\alpha} f\right)(t)$ as in Definition 2. Also, the convention $f^{(0)}(t)=f(t)$ leads to $\left({ }_{a}^{\mathrm{CFR}} \mathbf{D}^{\alpha} f\right)(t)=\left({ }_{a}^{\mathrm{CFR}} D^{\alpha} f\right)(t)$ and $\left({ }_{a}^{\mathrm{CFC}} \mathbf{D}^{\alpha} f\right)(t)=\left({ }_{a}^{\mathrm{CFC}} D^{\alpha} f\right)(t)$ for $0<\alpha \leq 1$.

Remark 1 In Definition 3, if we let $\alpha=n+1$ then $\beta=1$ and hence $\left({ }_{a}^{C F R} D^{\alpha} f\right)(t)=$ $\left.{ }_{a}^{C}{ }^{\mathrm{CFR}} \mathbf{D}^{1} f^{(n)}\right)(t)=f^{(n+1)}(t)$. Also, by noting that $\left({ }_{a}^{\mathrm{CF}} I^{1} f\right)(t)=\left({ }_{a} I^{1} f\right)(t)$, we see that for $\alpha=n+1$ we have $\left({ }_{a}^{C F} I^{\alpha} f\right)(t)=\left({ }_{a} I^{n+1} f\right)(t)$. Also, for $0<\alpha \leq 1$ we reobtain the concepts defined in Definition 2. Therefore, our generalization to the higher order case is valid.

Analogously, in the right case we have the following extension. 
Definition 4 Let $n<\alpha \leq n+1$ and $f$ be such that $f^{(n)} \in H^{1}(a, b)$. Set $\beta=\alpha-n$. Then $\beta \in(0,1]$ and we define

$$
\left({ }^{\mathrm{CFC}} \mathbf{D}_{b}^{\alpha} f\right)(t)=\left({ }^{\mathrm{CFC}} D_{b}^{\beta}(-1)^{n} f^{(n)}\right)(t) .
$$

In the right Riemann-Liouville sense it has the following form:

$$
\left({ }^{\mathrm{CFR}} \mathbf{D}_{b}^{\alpha} f\right)(t)=\left({ }^{\mathrm{CFR}} D_{b}^{\beta}(-1)^{n} f^{(n)}\right)(t) .
$$

The associated fractional integral is

$$
\left({ }^{\mathrm{CF}} \mathbf{I}_{b}^{\alpha} f\right)(t)=\left(I_{b}^{n \mathrm{CF}} I_{b}^{\beta} f\right)(t)
$$

The next proposition explains the action of the higher order integral operator ${ }_{a}^{\mathrm{CF}} \mathbf{I}^{\alpha}$ on the higher order $C F R$ and $C F C$ derivatives and vice versa, and the action of the CFR derivative on the $C F$ integral.

Proposition 1 For $u(t)$ defined on $[a, b]$ and $\alpha \in(n, n+1]$, for some $n \in \mathbb{N}_{0}$, we have:

- $\left({ }_{a}^{\mathrm{CFR}} \mathbf{D}_{a}^{\alpha \mathrm{CF}} \mathbf{I}^{\alpha} u\right)(t)=u(t)$.

- $\left({ }_{a}^{\mathrm{CF}} \mathbf{I}_{a}^{\alpha}{ }_{a}^{\mathrm{CFR}} \mathbf{D}^{\alpha} u\right)(t)=u(t)-\sum_{k=0}^{n-1} \frac{u^{(k)}(a)}{k !}(t-a)^{k}$.

- $\left({ }_{a}^{\mathrm{CF}} \mathbf{I}_{a}^{\alpha C F C} \mathbf{D}^{\alpha} u\right)(t)=u(t)-\sum_{k=0}^{n} \frac{u^{(k)}(a)}{k !}(t-a)^{k}$.

\section{Proof}

- By Definition 3 and the statement after Definition 2 we have

$$
\begin{aligned}
& \left({ }_{a}^{\mathrm{CFR}} \mathbf{D}_{a}^{\alpha}{ }_{a}^{\alpha F} \mathbf{I}^{\alpha} u\right)(t)=\left({ }_{a}^{\mathrm{CFR}} D^{\beta} \frac{d^{n}}{d t^{n}} a^{n}{ }_{a}^{n \mathrm{CF}} I^{\beta} u\right)(t) \\
& =\left({ }_{a}^{\mathrm{CFR}} D_{a}^{\beta \mathrm{CF}} I^{\beta} u\right)(t)=u(t),
\end{aligned}
$$

where $\beta=\alpha-n$.

- By Definition 3 and the statement after Definition 2 we have

$$
\begin{aligned}
\left({ }_{a}^{\mathrm{CF}} \mathbf{I}_{a}^{\alpha \mathrm{CFR}} \mathbf{D}^{\alpha} u\right)(t) & =\left({ }_{a} I_{a}^{n \mathrm{CF}} I_{a}^{\beta \mathrm{CFR}} D^{\beta} u^{(n)}\right)(t) \\
& ={ }_{a} I^{n} u^{(n)}(t)=u(t)-\sum_{k=0}^{n-1} \frac{u^{(k)}(a)}{k !}(t-a)^{k} .
\end{aligned}
$$

- By Lemma 1 applied to $f(t)=u^{(n)}(t)$ we have

$$
\begin{aligned}
\left({ }_{a}^{\mathrm{CF}} \mathbf{I}_{a}^{\alpha}{ }_{a}{ }^{C F C} \mathbf{D}^{\alpha} u\right)(t) & ={ }_{a} I_{a}^{n} I^{\beta}{ }_{a}{ }_{a} D^{\beta} u^{(n)}(t)={ }_{a} I^{n}\left[u^{(n)}(t)-u^{(n)}(a)\right] \\
& =u(t)-\sum_{k=0}^{n-1} \frac{u^{(k)}(a)}{k !}(t-a)^{k}-u^{(n)}(a) \frac{(t-a)^{n}}{n !} \\
& =u(t)-\sum_{k=0}^{n} \frac{u^{(k)}(a)}{k !}(t-a)^{k} .
\end{aligned}
$$

Similarly, for the right case we have the following. 
Proposition 2 For $u(t)$ defined on $[a, b]$ and $\alpha \in(n, n+1]$, for some $n \in \mathbb{N}_{0}$, we have:

- $\left({ }^{\mathrm{CFR}} \mathbf{D}_{b}^{\alpha \mathrm{CF}} \mathbf{I}_{b}^{\alpha} u\right)(t)=u(t)$.

- $\left({ }^{\mathrm{CF}} \mathbf{I}_{b}^{\alpha}{ }^{\mathrm{CFR}} \mathbf{D}_{b}^{\alpha} u\right)(t)=u(t)-\sum_{k=0}^{n-1} \frac{(-1)^{k} u^{(k)}(b)}{k !}(b-t)^{k}$.

- $\left({ }^{\mathrm{CF}} \mathbf{I}_{b}^{\alpha C F C} \mathbf{D}_{b}^{\alpha} u\right)(t)=u(t)-\sum_{k=0}^{n} \frac{(-1)^{k} u^{(k)}(b)}{k !}(b-t)^{k}$.

Example 1 Consider the initial value problem:

$$
\left({ }_{a}^{\mathrm{CFC}} \mathbf{D}^{\alpha} y\right)(t)=K(t), \quad t \in[a, b],
$$

where $K(t)$ is continuous on $[a, b]$. We consider two cases depending on the order $\alpha$.

- Assume $0<\alpha \leq 1, y(a)=c$ and $K(a)=0$. By applying ${ }_{a}^{\mathrm{CF}} \mathbf{I}^{\alpha}$ and making use of Proposition 1, we get the solution

$$
y(t)=c+\frac{1-\alpha}{B(\alpha)} K(t)+\frac{\alpha}{B(\alpha)} \int_{a}^{t} K(s) d s
$$

Notice that the condition $K(a)=0$ verifies that the initial condition $y(a)=c$. Also notice that when $\alpha \rightarrow 1$ we reobtain the solution of the ordinary initial value problem $y^{\prime}(t)=K(t), y(a)=c$.

- Assume $1<\alpha \leq 2, K(a)=0, y(a)=c_{1}, y^{\prime}(a)=c_{2}$. By applying ${ }_{a}^{\mathrm{CF}} \mathbf{I}^{\alpha}$ and making use of Proposition 1 and Definition 3 with $\beta=\alpha-1$, we get the solution

$$
y(t)=c_{1}+c_{2}(t-a)+\frac{2-\alpha}{B(\alpha-1)} \int_{a}^{t} K(s) d s+\frac{\alpha-1}{B(\alpha-1)} \int_{a}^{t}(t-s) K(s) d s
$$

Notice that the solution $y(t)$ verifies $y(a)=c_{1}$ without the use of $K(a)=0$. However, it verifies $y^{\prime}(a)=c_{2}$ under the assumption $K(a)=0$. Also, note that when $\alpha \rightarrow 2$ we reobtain the solution of the second order ordinary initial value problem $y^{\prime \prime}(t)=K(t)$.

In the next section, we prove existence and uniqueness theorems for some types of $C F C$ and CFR initial value problems.

Example 2 Consider the $C F C$ boundary value problem

$$
\left({ }_{a}^{\mathrm{CFC}} \mathbf{D}^{\alpha} y\right)(t)+q(t) y(t)=0, \quad 1<\alpha \leq 2, a<t<b, y(a)=y(b)=0 .
$$

Then $\beta=\alpha-1$ and by Proposition 1 applying the operator ${ }_{a}^{C F} I^{\alpha}$ will result in the solution

$$
y(t)=c_{1}+c_{2}(t-a)-\left({ }_{a}^{\mathrm{CF}} \mathbf{I}^{\alpha} q(\cdot) y(\cdot)\right)(t) .
$$

But $\left({ }_{a}^{\mathrm{CF}} \mathbf{I}^{\alpha} q(\cdot) y(\cdot)\right)(t)=\frac{1-\beta}{B(\beta)} \int_{a}^{t} q(s) y(s) d s+\frac{\beta}{B(\beta)} a I^{2} q(t) y(t)$. Hence, the solution has the form

$$
y(t)=c_{1}+c_{2}(t-a)-\frac{2-\alpha}{B(\alpha-1)} \int_{a}^{t} q(s) y(s) d s-\frac{\alpha-1}{B(\alpha-1)} \int_{a}^{t}(t-s) q(s) y(s) d s .
$$

The boundary conditions imply that $c_{1}=0$ and

$$
c_{2}=\frac{2-\alpha}{(b-a) B(\alpha-1)} \int_{a}^{b} q(s) y(s) d s+\frac{\alpha-1}{(b-a) B(\alpha-1)} \int_{a}^{b}(b-s) q(s) y(s) d s .
$$


Hence,

$$
\begin{aligned}
y(t)= & \frac{(2-\alpha)(t-a)}{(b-a) B(\alpha-1)} \int_{a}^{b} q(s) y(s) d s-\frac{(\alpha-1)(t-a)}{(b-a) B(\alpha-1)} \int_{a}^{b}(b-s) q(s) y(s) d s \\
& -\frac{2-\alpha}{B(\alpha-1)} \int_{a}^{t} q(s) y(s) d s-\frac{\alpha-1}{B(\alpha-1)} \int_{a}^{t}(t-s) q(s) y(s) d s .
\end{aligned}
$$

\section{Existence and uniqueness theorems for the initial value problem types}

In this section we prove existence uniqueness theorems for $A B C$ and $A B R$ type initial value problems.

Theorem 2 Consider the system

$$
\left({ }_{a}^{C F C} D^{\alpha} y\right)(t)=f(t, y(t)), \quad t \in[a, b], 0<\alpha \leq 1, y(a)=c
$$

such that $f(a, y(a))=0, A\left(\frac{1-\alpha}{B(\alpha)}+\frac{\alpha(b-a)}{B(\alpha)}\right)<1$, and $\left|f\left(t, y_{1}\right)-f\left(t, y_{2}\right)\right| \leq A\left|y_{1}-y_{2}\right|, A>0$. Here $f:[a, b] \times \mathbb{R} \rightarrow \mathbb{R}$ and $y:[a, b] \rightarrow \mathbb{R}$. Then the system (21) has a unique solution of the form

$$
y(t)=c+{ }_{a}^{\mathrm{CF}} I^{\alpha} f(t, y(t))
$$

Proof First, with the help of Proposition 1, (7) and taking into account that $f(a, y(a))=0$, it is straightforward to prove that $y(t)$ satisfies the system (21) if and only if it satisfies (22).

Let $X=\left\{x: \max _{t \in[a, b]}|x(t)|<\infty\right\}$ be the Banach space endowed with the norm $\|x\|=$ $\max _{t \in[a, b]}|x(t)|$. On $X$ define the linear operator

$$
(T x)(t)=c+{ }_{a}^{C F} I^{\alpha} f(t, x(t)) .
$$

Then, for arbitrary $x_{1}, x_{2} \in X$ and $t \in[a, b]$, we have by assumption

$$
\begin{aligned}
\left|\left(T x_{1}\right)(t)-\left(T x_{2}\right)(t)\right| & =||_{a}^{C F} I^{\alpha}\left[f\left(t, x_{1}(t)\right)-f\left(t, x_{2}(t)\right)\right] \mid \\
& \leq A\left(\frac{1-\alpha}{B(\alpha)}+\frac{\alpha(b-a)}{B(\alpha)}\right)\left\|x_{1}-x_{2}\right\|,
\end{aligned}
$$

and hence $T$ is a contraction. By the Banach contraction principle, there exists a unique $x \in X$ such that $T x=x$ and hence the proof is complete.

Remark 2 Similar existence and uniqueness theorems can be proved for the system (21) with higher order by making use of Proposition 1 . The condition $f(a, y(a))=0$ always cannot be avoided as we have seen in Example 1 with $f(t, y(t))=K(t)$. As a result of Theorem 2 we conclude that the fractional linear initial value problem

$$
\left({ }_{a}^{\mathrm{CFC}} D^{\alpha} y\right)(t)=\mu y(t), \quad \mu \in \mathbb{R}, t \in[a, b], 0<\alpha \leq 1, y(a)=c,
$$

only can have the trivial solution unless $\alpha=1$. Indeed, the solution satisfies $y(t)=c+$ $\mu \frac{1-\alpha}{B(\alpha)} y(t)+\frac{\alpha \mu}{B(\alpha)} \int_{a}^{t} y(s) d s$. This solution is only verified at $a$ if $(1-\alpha) y(a)=0$. 
Theorem 3 Consider the system

$$
\left({ }_{a}^{\mathrm{CFR}} D^{\alpha} y\right)(t)=f(t, y(t)), \quad t \in[a, b], 1<\alpha \leq 2, y(a)=c
$$

such that $\frac{A}{B(\alpha-1)}\left((2-\alpha)(b-a)+\frac{(\alpha-1)(b-a)^{2}}{2}\right)<1$ and $\left|f\left(t, y_{1}\right)-f\left(t, y_{2}\right)\right| \leq A\left|y_{1}-y_{2}\right|, A>0$. Also, $f:[a, b] \times \mathbb{R} \rightarrow \mathbb{R}$ and $y:[a, b] \rightarrow \mathbb{R}$. Then the system (21) has a unique solution of the form

$$
\begin{aligned}
y(t) & =c+{ }_{a}^{C F} \mathbf{I}^{\alpha} f(t, y(t)) \\
& =c+\frac{2-\alpha}{B(\alpha-1)} \int_{a}^{t} f(s, y(s)) d s+\frac{\alpha-1}{B(\alpha-1)}\left({ }_{a} I^{2} f(\cdot, y(\cdot))\right)(t) .
\end{aligned}
$$

Proof If we apply ${ }_{a}^{\mathrm{CF}} \mathbf{I}^{\alpha}$ to system (24) and make use of Proposition 1 with $\beta=\alpha-1$, then we obtain the representation (25). Conversely, if we apply ${ }_{a}^{C F R} \mathbf{D}^{\alpha}$, make use of Proposition 1 and note that

$$
{ }_{a}^{\mathrm{CFR}} \mathbf{D}^{\alpha}={ }_{a}^{\mathrm{CFR}} D^{\beta} \frac{d}{d t} c=0,
$$

we obtain the system (24). Hence, $y(t)$ satisfies the system (24) if and only if it satisfies (25).

Let $X=\left\{x: \max _{t \in[a, b]}|x(t)|<\infty\right\}$ be the Banach space endowed with the norm $\|x\|=$ $\max _{t \in[a, b]}|x(t)|$. On $X$ define the linear operator

$$
(T x)(t)=c+{ }_{a}^{C F} \mathbf{I}^{\alpha} f(t, x(t)) .
$$

Then, for arbitrary $x_{1}, x_{2} \in X$ and $t \in[a, b]$, we have by assumption

$$
\begin{aligned}
\left|\left(T x_{1}\right)(t)-\left(T x_{2}\right)(t)\right| & =\left.\right|_{a} ^{\mathrm{CF}} \mathbf{I}^{\alpha}\left[f\left(t, x_{1}(t)\right)-f\left(t, x_{2}(t)\right)\right] \mid \\
& \leq \frac{A}{B(\alpha-1)}\left((2-\alpha)(b-a)+\frac{(\alpha-1)(b-a)^{2}}{2}\right)\left\|x_{1}-x_{2}\right\|,
\end{aligned}
$$

and hence $T$ is a contraction. By the Banach contraction principle, there exists a unique $x \in X$ such that $T x=x$ and hence the proof is complete.

\section{The Lyapunov inequality for the CFR boundary value problem}

In this section, we prove a Lyapunov inequality for an CFR boundary value problem of order $2<\alpha \leq 3$.

Consider the boundary value problem

$$
\left({ }_{a}^{\mathrm{CFR}} \mathbf{D}^{\alpha} y\right)(t)+q(t) y(t)=0, \quad 2<\alpha \leq 3, t \in(a, b), y(a)=y(b)=0 .
$$

Lemma $2 y(t)$ is a solution of the boundary value problem (27) if and only if it satisfies the integral equation

$$
y(t)=\int_{a}^{b} G(t, s) T(s, y(s)) d s,
$$


where

$$
G(t, s)=\left\{\begin{array}{ll}
\frac{(t-a)(b-s)}{b-a}, & a \leq t \leq s \leq b, \\
\left(\frac{(t-a)(b-s)}{b-a}-(t-s)\right), & a \leq s \leq t \leq b,
\end{array}\right\}
$$

and

$$
T(t, y(t))=\left({ }_{a}^{\mathrm{CF}} \mathbf{I}^{\beta} q(\cdot) y(\cdot)\right)(t)=\frac{1-\beta}{B(\beta)} q(t) y(t)+\frac{\beta}{B(\beta)}\left({ }_{a} I^{1} q(\cdot) y(\cdot)\right)(t), \quad \beta=\alpha-2 .
$$

Proof Apply the integral ${ }_{a}^{\mathrm{CF}} \mathbf{I}^{\alpha}$ to (27) and make use of Definition 3 and Proposition 1 with $n=2$ and $\beta=\alpha-2$ to obtain

$$
\begin{aligned}
y(t) & =c_{1}+c_{2}(t-a)-\left(a^{2} T(\cdot, y(\cdot))\right)(t) \\
& =c_{1}+c_{2}(t-a)-\int_{a}^{t}(t-s) T(s, y(s)) d s .
\end{aligned}
$$

The condition $y(a)=0$ implies that $c_{1}=0$ and the condition $y(b)=0$ implies that $c_{2}=$ $\frac{1}{b-a} \int_{a}^{b}(b-s) T(s, y(s)) d s$ and hence

$$
y(t)=\frac{t-a}{b-a} \int_{a}^{b}(b-s) T(s, y(s)) d s-\int_{a}^{t}(t-s) q(s) T(s, y(s)) d s .
$$

Then the result follows by splitting the integral

$$
\int_{a}^{b}(b-s) T(s, y(s)) d s=\int_{a}^{t}(b-s) T(s, y(s)) d s+\int_{t}^{b}(b-s) T(s, y(s)) d s .
$$

Lemma 3 The Green's function $G(t, s)$ defined in Lemma 2 has the following properties:

- $G(t, s) \geq 0$ for all $a \leq t, s \leq b$.

- $\max _{t \in[a, b]} G(t, s)=G(s, s)$ for $s \in[a, b]$.

- $H(s, s)$ has a unique maximum, given by

$$
\max _{s \in[a, b]} G(s, s)=G\left(\frac{a+b}{2}, \frac{a+b}{2}\right)=\frac{(b-a)}{4} .
$$

\section{Proof}

- It is clear that $g_{1}(t, s)=\frac{(t-a)(b-s)}{b-a} \geq 0$. Regarding the part $g_{2}(t, s)=\left(\frac{(t-a)(b-s)}{b-a}-(t-s)\right)$ we see that $(t-s)=\frac{t-a}{b-a}\left(b-\left(a+\frac{(s-a)(b-a)}{(t-a)}\right)\right)$ and that $a+\frac{(s-a)(b-a)}{(t-a)} \geq s$ if and only if $s \geq a$. Hence, we conclude that $g_{2}(t, s) \geq 0$ as well. Hence, the proof of the first part is complete.

- Clearly, $g_{1}(t, s)$ is an increasing function in $t$. Differentiating $g_{2}$ with respect to $t$ for every fixed $s$ we see that $g_{2}$ is a decreasing function in $t$.

- Let $g(s)=G(s, s)=\frac{(s-a)(b-s)}{b-a}$. Then one can show that $g^{\prime}(s)=0$ if $s=\frac{a+b}{2}$ and hence the proof is concluded by verifying that $g\left(\frac{a+b}{2}\right)=\frac{b-a}{4}$.

In the next lemma, we estimate $T(t, y(t))$ for a function $y \in C[a, b]$.

Lemma 4 For $y \in C[a, b]$ and $2<\alpha \leq 3, \beta=\alpha-2$, we have for any $t \in[a, b]$

$$
|T(t, y(t))| \leq R(t)\|y\|
$$


where

$$
R(t)=\left[\frac{3-\alpha}{B(\alpha-2)}|q(t)|+\frac{\alpha-2}{B(\alpha-2)} \int_{a}^{t}|q(s)| d s\right] .
$$

Theorem 4 If the boundary value problem (27) has a nontrivial solution, where $q(t)$ is a real-valued continuous function on $[a, b]$, then

$$
\int_{a}^{b} R(s) d s>\frac{4}{b-a}
$$

Proof Assume $y \in Y=C[a, b]$ is a nontrivial solution of the boundary value problem (27), where $\|y\|=\sup _{t \in[a, b]}|y(t)|$. By Lemma 2, $y$ must satisfy

$$
y(t)=\int_{a}^{b} G(t, s) T(s, y(s)) d s .
$$

Then, by using the properties of the Green's function $G(t, s)$ proved in Lemma 3 and Lemma 4, we come to the conclusion that

$$
\|y\|<\frac{b-a}{4} \int_{a}^{b} R(s) d s\|y\| .
$$

From this (30) follows.

Remark 3 Note that if $\alpha \rightarrow 2^{+}$, then $R(t)$ tends to $|q(t)|$ and hence one obtains the classical Lyapunov inequality (8).

Example 3 Consider the following CFR Sturm-Liouville eigenvalue problem (SLEP) of order $2<\alpha \leq 3$ :

$$
\left({ }_{0}^{\mathrm{CFR}} \mathbf{D}^{\alpha} y\right)(t)+\lambda y(t)=0, \quad 0<t<1, y(0)=y(1)=0 .
$$

If $\lambda$ is an eigenvalue of (31), then by Theorem 4 with $q(t)=\lambda$, we have

$$
\begin{aligned}
T(t) & =\left[\frac{3-\alpha}{B(\alpha-2)}|\lambda|+\frac{\alpha-2}{B(\alpha-2)}\left({ }_{0} I^{1}|\lambda|\right)(t)\right] \\
& =|\lambda|\left[\frac{3-\alpha}{B(\alpha-2)}+\frac{\alpha-2}{B(\alpha-2)} t\right] .
\end{aligned}
$$

Hence, we must have

$$
\int_{0}^{1} T(s) d s=|\lambda|\left[\frac{3-\alpha}{B(\alpha-2)}+\frac{\alpha-2}{2 B(\alpha-2)}\right]>4
$$

Hence,

$$
|\lambda|>4\left[\frac{3-\alpha}{B(\alpha-2)}+\frac{\alpha-2}{2 B(\alpha-2)}\right]^{-1}
$$


Notice that the limiting case $\alpha \rightarrow 2^{+}$implies that $|\lambda|>4$. This is the lower bound for the eigenvalues of the ordinary eigenvalue problem:

$$
y^{\prime \prime}(t)+\lambda y(t)=0, \quad 0<t<1, y(0)=y(1)=0 .
$$

\section{Conclusions}

Fractional derivatives and their corresponding integral operators are of importance in modeling various problems in engineering, science and medicine. To provide the researchers with the possibility of modeling by means of higher order arbitrary dynamical systems we extended fractional calculus whose derivatives depend on nonsingular exponential function kernels to higher order. The corresponding higher order integral operators have been defined as well and confirmed. The right fractional extension is also considered. To set up the basic concepts we proved existence and uniqueness theorems by means of the Banach fixed point theorem for initial value problems in the frame of $C F C$ and CFR derivatives. We realized that the condition $f(a, y(a))=0$ is necessary to guarantee a unique solution and hence the fractional linear initial value problem with constant coefficients results in the trivial solution unless the order is a positive integer. We used our extension to higher order to prove a Lyapunov type inequality for a CFR boundary value problem with order $2<\alpha \leq 3$ and then obtained the classical ordinary case when $\alpha$ tends to 2 from the right. This proves a different behavior from the classical fractional case, where the Lyapunov inequality was proved for a fractional boundary problem of order $1<\alpha \leq 2$ and the classical ordinary case was verified when $\alpha$ tends to 2 from left. In connection to this behavior, we propose the following open problem: Is it possible to formulate a sequential CFR boundary value problem whose Green's function is so nice as to prove a Lyapunov type inequality?

\section{Acknowledgements}

The author would like to thank Prince Sultan University for funding this work through research group Nonlinear Analysis Methods in Applied Mathematics (NAMAM) group number RG-DES-2017-01-17.

\section{Competing interests}

The authors declare that they have no competing interests.

\section{Author's contributions}

The author read and approved the final manuscript.

\section{Publisher's Note}

Springer Nature remains neutral with regard to jurisdictional claims in published maps and institutional affiliations.

Received: 9 May 2017 Accepted: 18 July 2017 Published online: 06 October 2017

\section{References}

1. Samko, G, Kilbas, AA, Marichev, Ol: Fractional Integrals and Derivatives: Theory and Applications. Gordon \& Breach, Yverdon (1993)

2. Podlubny, I: Fractional Differential Equations. Academic Press, San Diego (1999)

3. Kilbas, A, Srivastava, MH, Trujillo, JJ: Theory and Application of Fractional Differential Equations. Mathematics Studies, vol. 204. North-Holland, Amsterdam (2006)

4. Tenreiro Machado, JA, Kiryakova, V, Mainardi, F: A poster about the recent history of fractional calculus. Fract. Calc Appl. Anal. 13(3), 329-334 (2010)

5. Tenreiro Machado, JA: Fractional dynamics of a system with particles subjected to impacts. Commun. Nonlinear Sci. Numer. Simul. 16(12), 4596-4601 (2011)

6. Baleanu, D, Diethelm, K, Scalas, E, Trujillo, JJ: Fractional Calculus: Models and Numerical Methods, 2nd edn. (2016)

7. Bozkurt, F, Abdeljawad, T, Haiji, MA: Stability analysis of a fractional order differential equation model of a brain tumor growth depending on the density. Appl. Comput. Math. 14(1), 50-62 (2015)

8. Caputo, M, Fabrizio, M: A new definition of fractional derivative without singular kernal. Prog. Fract. Differ. Appl. 1(2), 73-85 (2015) 
9. Losada, J, Nieto, JJ: Properties of a new fractional derivative without singular kernal. Prog. Fract. Differ. Appl. 1(2), 87-92 (2015)

10. Atangana, A, Baleanu, D: Caputo-Fabrizio derivative applied to groundwater flow within confined aquifer. J. Eng. Mech. 143(5), Article ID D4016005 (2017)

11. Baleanu, D, Mousalou, A, Rezapour, S: A new method for investigating approximate solutions of some fractional integro-differential equations involving the Caputo-Fabrizio derivative. Adv. Differ. Equ. 2017. Article ID 51 (2017)

12. Gomez-Aguilar, JF, Baleanu, D: Schrodinger equation involving fractional operators with non-singular kernel. J. Electromagn. Waves Appl. 31(7), 752-761 (2017)

13. Atangana, A, Baleanu, D: New fractional derivative with non-local and non-singular kernal. Therm. Sci. 20(2), 757-763 (2016)

14. Abdeljawad, T, Baleanu, D: Integration by parts and its applications of a new nonlocal fractional derivative with Mittag-Leffler nonsingular kernel. J. Nonlinear Sci. Appl. 10(3), 1098-1 107 (2017)

15. Abdeljawad, T, Baleanu, D: On fractional derivatives with exponential kernel and their discrete versions. J. Rep. Math. Phys. 80(1), 11-27 (2017)

16. Abdeljawad, T, Baleanu, D: Discrete fractional differences with nonsingular discrete Mittag-Leffler kernels. Adv. Differ. Equ. 2016, Article ID 232 (2016). doi:10.1186/s13662-016-0949-5

17. Abdeljawad, T, Baleanu, D: Monotonicity results for fractional difference operators with discrete exponential kernels. Adv. Differ. Equ. 2017, Article ID 78 (2017). doi:10.1186/s13662-017-1126-1

18. Abdeljawad, T, Baleanu, D: Monotonicity results for a nabla fractional difference operator with discrete Mittag-Leffler kernels. Chaos Solitons Fractals (2017). doi:10.1016/j.chaos.2017.04.006

19. Baleanu, D, Abdeljawad, T, Jarad, F: Fractional variational principles with delay. J. Phys. A, Math. Theor. 41(31), 315403 (2008)

20. Jarad, F, Abdeljawad, T, Baleanu, D: Fractional variational principles with delay within Caputo derivatives. Rep. Math. Phys. 65, 17-28 (2010)

21. Lyapunov, AM: Probleme général de la stabilité du mouvement. Ann. Fac. Sci. Univ. Toulouse 2, 27-247 (1907). Reprinted in: Ann. Math. Studies, No. 17, Princeton (1947)

22. Ferreira, RAC: A Lyapunov-type inequality for a fractional boundary value problem. Fract. Calc. Appl. Anal. 6(4), 978-984 (2013)

23. Chdouh, A, Torres, DFM: A generalized Lyapunov's inequality for a fractional boundary value problem. J. Comput. Appl. Math. 312, 192-197 (2017)

24. Jeli, M, Samet, B: Lyapunov-type inequalities for fractional boundary value problems. Electron. J. Differ. Equ. 2015 Article ID 88 (2015)

25. O'Regan, D, Samet, B: Lyapunov-type inequalities for a class of fractional differential equations. J. Inequal. Appl. 2015, Article ID 247 (2015)

26. Rong, J, Bai, C: Lyapunov-type inequality for a fractional differential equation with fractional boundary conditions. Adv. Differ. Equ. 2015, Article ID 82 (2015)

27. Ferreira, RAC: Some discrete fractional Lyapunov-type inequalities. Fract. Differ. Calc. 5(1), 87-92 (2015)

28. Jleli, M, Samet, B: A Lyapunov-type inequality for a fractional $q$-difference boundary value problem. J. Nonlinear Sci. Appl. 9, 1965-1976 (2016)

29. Abdeljawad, T: A Lyapunov type inequality for fractional operators with nonsingular Mittag-Leffler kernel. J. Inequal. Appl. 2017, Article ID 130 (2017). doi:10.1186/s13660-017-1400-5

30. Abdeljawad, T, Al-Mdallal, QM, Hajii, MA: Arbitrary order fractional difference operators with discrete exponential kernels and applications. Discrete Dyn. Nat. Soc. 2017, Article ID 4149320 (2017)

31. Abdeljawad, T, Madjidi, F: Lyapunov-type inequalities for fractional difference operators with discrete Mittag-Leffler kernel of order $2<\alpha<5 / 2$. Eur. Phys. J. Spec. Top. (2017, to appear)

\section{Submit your manuscript to a SpringerOpen ${ }^{\circ}$ journal and benefit from:}

- Convenient online submission

- Rigorous peer review

- Open access: articles freely available online

- High visibility within the field

- Retaining the copyright to your article

Submit your next manuscript at $\gg$ springeropen.com 